\title{
Differentiating Carotid Terminus Occlusions into Two Distinct Populations Based on Willisian Collateral Status
}

\author{
Sun-Uk Lee, ${ }^{\mathrm{a}}$ Ji Man Hong, ${ }^{\mathrm{a}}$ Sun Yong Kim, ${ }^{\mathrm{b}}$ Oh Young Bang, ${ }^{\mathrm{c}}$ Andrew M. Demchuk, ${ }^{\mathrm{d}}$ Jin Soo Lee ${ }^{\mathrm{a}}$ \\ aDepartment of Neurology, Ajou University School of Medicine, Ajou University Medical Center, Suwon, Korea \\ 'Department of Radiology, Ajou University School of Medicine, Ajou University Medical Center, Suwon, Korea \\ 'Department of Neurology, Samsung Medical Center, Sungkyunkwan University, Seoul, Korea \\ ${ }^{d}$ Calgary Stroke Program, Departments of Clinical Neurosciences and Radiology, Hotchkiss Brain Institute, University of Calgary, Calgary, AB, Canada
}

Background and Purpose The outcomes of acute internal carotid artery (ICA) terminus occlusions are poor. We classified ICA terminus occlusions into 2 groups according to the occlusion pattern of the circle of Willis and hypothesized that clinical outcomes would significantly differ between them. Methods Consecutive patients with acute ICA terminus occlusions evaluated by baseline computed tomographic angiography were enrolled. We investigated the occlusion patterns in the circle of Willis, retrospectively classified patients into simple ICA terminus occlusion (STO; with good Willisian collaterals from neighboring cerebral circulation) and complex ICA terminus occlusion (CTO; with one or more of A2 anterior cerebral artery, fetal posterior cerebral artery occlusion, or hypoplastic/absent contralateral A1; or with poor collaterals from anterior communicating artery) groups, and compared their baseline characteristics and outcomes.

Results The STO group $(n=58)$ showed smaller infarct volumes at 72 hours than the CTO group $(\mathrm{n}=34)$ (median, $81 \mathrm{~mL}$ [interquartile range, 38-192] vs. $414 \mathrm{~mL}$ [193-540], $P<0.001$ ) and more favorable outcomes (3-month modified Rankin Scale $0-3,44.8 \%$ vs. 8.8\%, $P<0.001$; 3-month mortality, $24.1 \%$ vs. $67.6 \%, P<0.001$ ). In multivariable analyses, STO remained an independent predictor for favorable outcomes (odds ratio 6.1, $P=0.010$ ).

Conclusions Favorable outcomes in STO group suggested that the outcomes of acute ICA terminus occlusions depend on Willisian collateral status. Documenting the subtypes on computed tomographic angiography would help predict patient outcome.

Keywords Cerebral infarction; Carotid artery thrombosis; Endovascular procedures; Circle of Willis; Collateral circulation

\author{
Correspondence: Jin Soo Lee \\ Department of Neurology, Ajou \\ University School of Medicine, Ajou \\ University Hospital, 164 Worldcup-ro, \\ Yeongtong-gu, Suwon 16499, Korea \\ Tel: +82-31-219-5175 \\ Fax: +82-31-219-5178 \\ E-mail: jinsoo22@gmail.com
}

Received: October 6, 2015

Revised: January 7, 2016

Accepted: January 18, 2016

This work was partly supported by the Basic Science Research Program through the National Research Foundation of Korea (NRF), funded by the Ministry of Education (NRF-2014R1A1A1008249).

The authors have no financial conflicts of interest.

\section{Introduction}

Acute internal carotid artery (ICA) terminus occlusions frequently lead to adverse neurological outcomes and are associated with high mortality. ${ }^{1-3}$ The poor prognosis of ICA terminus occlusions may be partly due to the large thrombus burden precluding sufficient recanalization. ICA terminus occlusions are rarely recanalized with intravenous (IV) thrombolytic therapy alone, and adjunctive endovascular treatment has been proposed as more appropriate to achieve improved recanalization. ${ }^{1.4}$ Prior studies on intra-arterial (IA) thrombolytics found that while they resulted in a substantial recanalization rate, they were associated with a high rate of hemorrhagic transformation and low rates of good clinical outcomes, similar to IV treatment alone., ${ }^{2,5}$ One study found that mechanical thrombectomy devices were more effective than IA urokinase infusion for recanalization, but they 
still led to a high rate of poor outcomes. ${ }^{3}$

In general, patients with abundant leptomeningeal collaterals show better clinical outcomes and lower risk of hemorrhagic transformation, and abundant collaterals have been shown to limit the initial infarct core size and subsequent infarct growth. ${ }^{6.7}$ The role of collaterals may be unique in ICA terminus occlusions due to the importance of Willisian collateral status. To date, there have been few reports of systematic approaches to evaluate Willisian collateral status for clinicians faced with ICA terminus occlusions considering revascularization treatment., ${ }^{89}$

In the present study, we hypothesized that the presence or absence of Willisian collaterals in patients with acute ICA terminus occlusion would significantly influence clinical outcomes. We analyzed the occlusion patterns and anatomical variations within the circle of Willis by baseline computed tomographic angiography (CTA) and propose an imaging classification that could serve as a predictive marker for acute ICA terminus occlusion patients.

\section{Methods}

\section{Patient enrollment}

We enrolled patients with acute ischemic stroke and ICA occlusions from a consecutively registered database from March 2006 to August 2013 at a university hospital. The diagnosis of acute ICA occlusions was based on CTA including intracranial and extracranial arteries. Patients with acute symptomatic ICA terminus occlusion, which was presented with T-type or L-type occlusions, were included. A T-type ICA occlusion was defined when (1) the terminal segment (C7) of the ICA was not visible, and there was occlusion of (2) the M1 segment in the middle cerebral artery and (3) the ipsilateral A1 segment in the anterior cerebral artery. ${ }^{4,10}$ Sparing of the ipsilateral A1 segment due to agenesis represented an L-type ICA occlusion. Patients with isolated proximal ICA occlusions, those with isolated ICA terminal occlusions with patent ipsilateral $\mathrm{A} 1$ and $\mathrm{M} 1$ segments and those with concomitant distant tandem occlusions such as distal M1, M2, or A2 segments were excluded. Patients whose onset-to-CTA time was $>6$ hours and whose CTA was not available were also excluded.

\section{Protocols}

We followed a standard treatment algorithm for acute intracranial large artery occlusion management, which included bridging endovascular treatment with IV recombinant tissue plasminogen activator (rtPA) when patients were eligible for thrombolysis. Patients who met the criteria for IV rtPA within 3-4.5 hours were treated with $0.9 \mathrm{mg} / \mathrm{kg}$ IV rtPA. If a major cerebral artery corresponding to stroke signs was occluded on CTA, the patients were brought to the angiography suite. Patients were considered candidates for endovascular treatment if the onset-to-puncture time was expected to be $\leq 6$ hours. The methods of endovascular treatment were at the treating physicians' discretion. IA fibrinolysis was mainly performed prior to 2010, and mechanical thrombectomy, such as clot aspiration with the Penumbra system (Penumbra, Alameda, CA) and embolus retrieval with the Solitaire stent system (Covidien, Irvine, CA), was mainly used after 2011. The procedure time was defined as the time between the first working projection image and final angiography following treatment of the target occluded artery. Informed consent for undertaking IV rtPA or IA revascularization treatment was obtained from all patients or their relatives, and the study was approved by the local institutional review board.

\section{Classification of ICA terminus occlusion}

We investigated the involvement of each major cerebral artery and divided the patients into simple ICA terminus occlusion (STO) and complex ICA terminus occlusion (CTO) groups based on the occlusion pattern obtained from CTA (Figure 1).

The STO group was classified by acute ICA terminus occlusion and patency of not only the ipsilateral A2 segment via the anterior communicating artery but also the ipsilateral posterior cerebral artery via the posterior communicating artery or the P1 segment.

The CTO group was classified by acute ICA terminus occlusion and had one or more of the following:

1) occlusion of the ipsilateral $A 2$ or more distal segment

2) occlusion of the fetal-type ipsilateral posterior cerebral artery (C6 segment of ICA)

3) insufficient contralateral Willisian collateral blood supply via the anterior communicating artery due to contralateral agenesis of A1 (absent or severely hypoplastic)

4) contralateral ICA occlusion

\section{Clinical and imaging parameters}

The neurological status of each patient was evaluated using the National Institute of Health Stroke Scale (NIHSS) score at admission and discharge. ${ }^{11}$ Patients who died were assigned an NIHSS score of $42 .^{12}$ Stroke etiology was determined according to the Trial of Org 10172 in Acute Stroke Treatment classification. ${ }^{13}$ Prognosis was defined as favorable when a patient had a modified Rankin Scale score of 0-3 at 3 months. The 3-month mortality rate was also examined.

Computed tomography (CT) scans (including non-contrast CT [NCCT], CTA, and phase-contrast CT [PCCT]; SOMATOM Sensation 16, Siemens, Erlangen, Germany) were initially obtained for each patient upon admission to the emergency department. NCCT and PCCT were performed using the axial technique with 
Simple ICA terminus occlusions

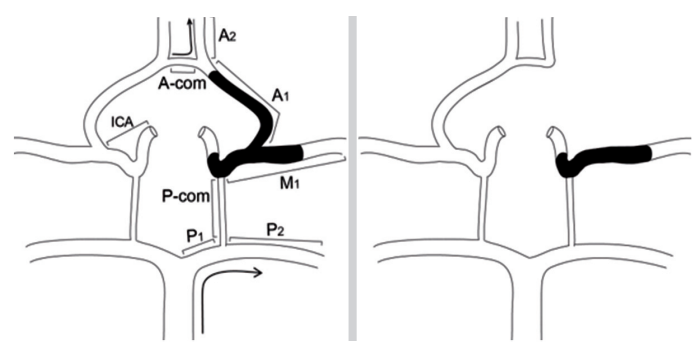

Simple T type

L type

A

Complex ICA terminus occlusions

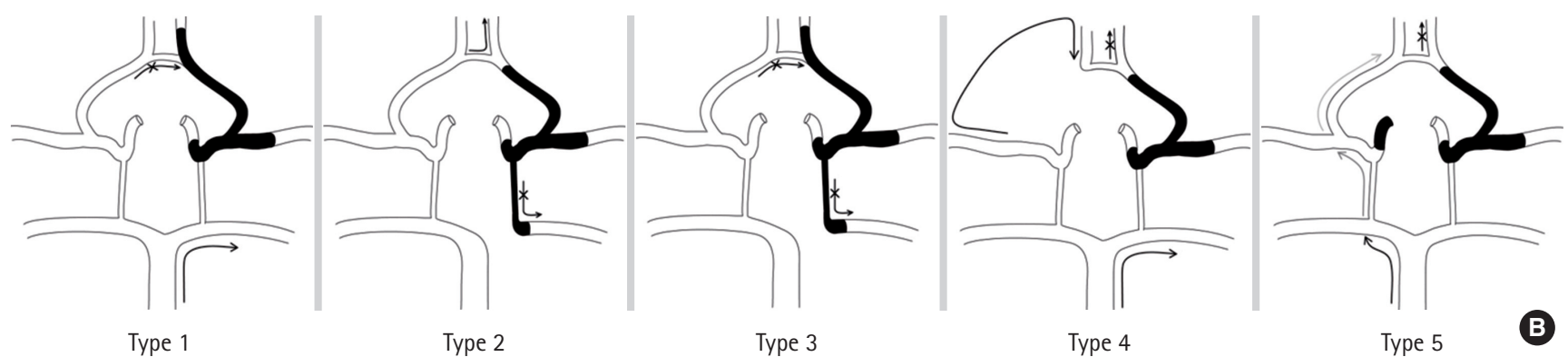

Figure 1. Simple and complex internal carotid artery (ICA) terminus occlusions. (A) Simple ICA terminus occlusion: the thrombus is confined to the bifurcation of the ICA, ipsilateral $M 1 \pm A 1$. The ipsilateral $A 2$ is irrigated by the collateral flow via the anterior communicating artery (A-com). (B) Complex ICA terminus occlusions. Type 1: the thrombus extends beyond the ipsilateral $A 1$ to involve the $A 2$, or agenesis of the anterior communicating artery halts the anterior cerebral artery leptomeningeal collaterals. Type 2: the ipsilateral posterior cerebral artery is also occluded by the distal ICA occlusion. Type 3: combination of types 1 and 2. Type 4: absence of the contralateral A1. Type 5: occlusion of the contralateral ICA. P-com, posterior communicating artery.

$120 \mathrm{kVp}, 270 \mathrm{~mA}$, and 5-mm section thickness reconstructions. CTA was performed by scanning from the vertex to the aortic arch with the following parameters: 0.5 s/rotation; pitch, 1.3; collimation, $0.75 \mathrm{~mm}$; maximal $\mathrm{mA}, 170$; $\mathrm{kVp}, 100$; and field of view, $220 \mathrm{~mm}$. Nonionic contrast material (80-100 mL) was administered by a power injector at 4-5 mL/s into an antecubital vein with a semi-automatic contrast bolus triggering technique. The CTA source images were post-processed to create coronal, sagittal, and axial multiplanar reformats in maximum intensity projection images and volume-rendered 3D images. PCCT was initiated after a 13-second delay from the end of CTA.

Imaging parameters were retrospectively evaluated as follows. Low attenuated tissue regions were evaluated by the NCCT Alberta Stroke Program Early CT Score (ASPECTS) ${ }^{14}$ and PCCT-ASPECTS. The degree of arterial and arteriolar circulation was scored on each ASPECTS-corresponding area on maximum intensity projection axial images of the CTA (CTA-col-ASPECTS) and PCCT (PCCTCol-ASPECTS) to evaluate the degree of collaterals, the scale of which was modified from a previous study. ${ }^{15}$ Hemorrhagic transformation was determined according to the European Cooperative Acute Stroke Study ${ }_{1}^{16}$ with NCCT performed immediately after endovascular treatment and up to 48-72 hours after stroke onset if clinical deterioration was noted. Infarct volume was measured at 2-3 days post-admission by standard follow-up imaging and determined using the total volume of diffusion restriction lesions observed on magnetic resonance imaging or, when unavailable, from the total hypodensity observed on CT performed during the same period. The infarct volume was calculated using a previously described semi-quantitative method. ${ }^{17}$ The degree of reperfusion was quantified according to the modified Thrombolysis in Cerebral Infarction classification, ${ }^{12,18}$ and successful reperfusion was defined as 2b-3. Two experienced neurologists (SUL, JSL) blinded to patients' clinical information, reviewed the imaging data.

\section{Statistical analysis}

Statistical analyses were performed using SPSS (version 22.0; IBM SPSS, Chicago, IL). Parameters were compared with the $\chi^{2}$ and Mann-Whitney $\mathrm{U}$ tests for non-parametric variables, and $t$ test for continuous variables. Multivariable logistic regression analyses were performed to identify STO as an independent factor for favorable outcomes after adjusting for age, sex, NIHSS score, and diabetes mellitus in model 1. Model 2 was analyzed in patients who had endovascular treatment and successful reperfusion (Thrombolysis In Cerebral Infarction grade $2 b$ and 3 ) was also adjusted. 


\section{Results}

\section{General demographics}

Ninety-two patients were included in the study after excluding patients with proximal carotid occlusion with patent terminal segment $(n=42)$; isolated ICA terminal segment occlusion with patent ipsilateral $A 1$ and $M 1$ segments $(n=39)$; tandem occlusion in distal M1, M2, or A2 $(n=24)$; CTA performed after 6 hours ( $n=17)$; or incomplete CTA evaluation $(n=6)$ (Figure 2). The demographic and baseline characteristics of the patients are listed in Table 1. Among the included patients, 34 (37\%) were classi-

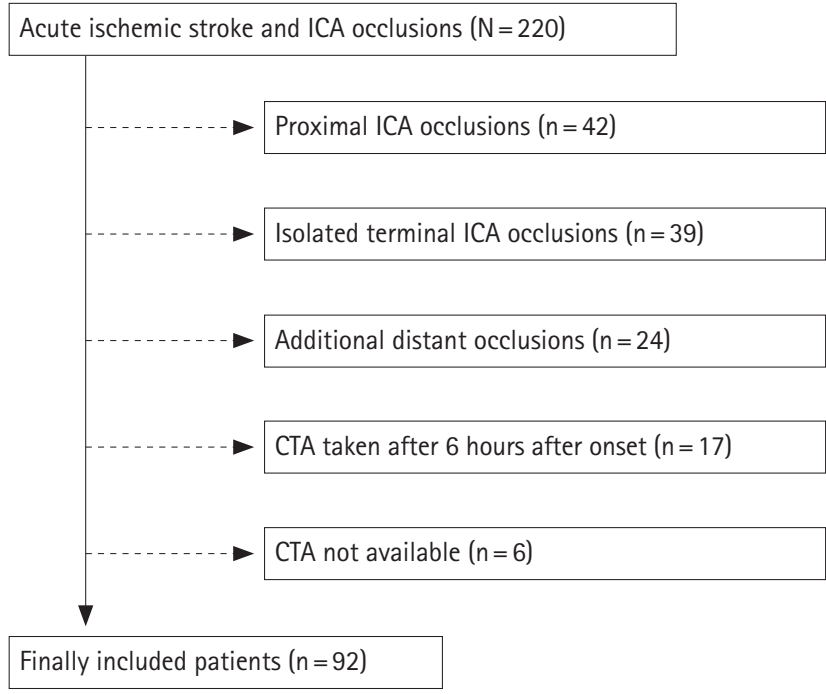

Figure 2. Flow diagram of the current study. fied as having CTO. In the СTO group, 9 patients were type $1 ; 10$ patients, type 2; 3 patients, type 3; 9 patients, type 4; and 3 patients, type 5. Patients in the STO occlusion group were younger $(67.7 \pm 13.6$ vs. $73.3 \pm 10.9$ years, $P=0.044)$, whereas sex distribution, vascular risk factors, and stroke etiology did not differ between the groups. However, NIHSS score on admission was significantly higher in the CTO group (median, 16 vs. $19, P=0.001$ ).

\section{Initial ASPECTS and collateral assessment on CT modalities}

The ASPECTS values on NCCT and PCCT were significantly lower in the CTO group (median, 5 vs. 3.5, $P=0.005$ and 6 vs. 3 , $P<0.001$, respectively), as were the CTA-col-ASPECTS and PCCTcol-ASPECTS values (median, 4 vs. 1 and 8 vs. 3, respectively; both $P<0.001)$.

\section{Revascularization treatments and outcomes}

The frequencies of IV rtPA infusion (58.6\% vs. $52.9 \%, P=0.596$ ) and endovascular treatment $(67.2 \%$ vs. $79.4 \%, P=0.211)$ did not differ between the STO and CTO groups, nor did the frequency of patients in whom revascularization treatment was not attempted (29.3\% vs. $20.6 \%, P=0.463$ ) and the onset-to-final angiography time (344 minutes vs. 325 minutes, $P=0.447$ ) (Table 1).

Despite the similar treatment patterns, imaging and clinical outcomes differed between the groups (Table 2). The frequency of successful reperfusion was higher in the STO group (46.5\% vs. $25.9 \%, P=0.085)$, whereas the occurrence of parenchymal he-

Table 1. Baseline characteristics and treatment modalities of the study patients

\begin{tabular}{|c|c|c|c|}
\hline & STO & СТО & $P$ \\
\hline Number & 58 & 34 & \\
\hline Mean age (year) & $67.7 \pm 13.6$ & $73.3 \pm 10.9$ & 0.044 \\
\hline Sex, male $(\%)$ & $35(60.3)$ & $16(47.1)$ & 0.216 \\
\hline Diabetes mellitus (\%) & $11(19.0)$ & $4(11.8)$ & 0.560 \\
\hline Hypertension (\%) & $37(63.8)$ & 25 (73.5) & 0.336 \\
\hline Hyperlipidemia (\%) & $37(63.8)$ & $18(52.9)$ & 0.306 \\
\hline Atrial fibrillation $(\%)$ & $38(65.5)$ & $27(79.4)$ & 0.158 \\
\hline Previous stroke $(\%)$ & $13(22.4)$ & $7(20.6)$ & 0.838 \\
\hline Current smoker (\%) & $11 / 57$ (19.3) & $3 / 32(9.4)$ & 0.363 \\
\hline Antiplatelet therapy at stroke onset (\%) & $9(15.5)$ & $6(17.6)$ & 0.790 \\
\hline Anticoagulant therapy at stroke onset (\%) & $4(6.9)$ & $8(23.5)$ & 0.029 \\
\hline Median NIHSS on admission (IOR) & $16(14-19)$ & $19(17-22)$ & 0.001 \\
\hline Median ASPECTS (IOR) & $5(3-8)$ & $3.5(2-7)$ & 0.005 \\
\hline Median PCCT ASPECTS (IOR) & $6(3-8)$ & $3(1-5)$ & $<0.001$ \\
\hline Median CTA-col-ASPECTS (IQR) & $4(3-6)$ & $1(0-3)$ & $<0.001$ \\
\hline Median PCCT-col-ASPECTS (IOR) & $8(6-9)$ & $3(2-5)$ & $<0.001$ \\
\hline Intravenous rtPA, n (\%) & $34(58.6)$ & $18(52.9)$ & 0.596 \\
\hline Endovascular treatment, $\mathrm{n}(\%)$ & $39(67.2)$ & $27(79.4)$ & 0.211 \\
\hline No revascularization treatment, $\mathrm{n}(\%)$ & $17(29.3)$ & $7(20.6)$ & 0.463 \\
\hline
\end{tabular}

STO, simple internal carotid artery terminus occlusion; CTO, complex internal carotid artery terminus occlusion; NIHSS, National Institute of Health Stroke Scale; IQR, interquartile range; ASPECTS, Alberta Stroke Program Early CT Score; PCCT, post-contrast axial parenchymal images; CTA, computed tomographic angiography; CTA/PCCT-col-ASPECTS, ASPECTS corresponding area on maximum intensity projection axial images of CTA/PCCT; rtPA, recombinant tissue plasminogen activator. 
Table 2. Imaging and clinical outcomes

\begin{tabular}{|c|c|c|c|}
\hline & STO & СТО & $P$ \\
\hline Mean onset-to-CT time (minute) & $121.9 \pm 95.6$ & $112.6 \pm 71.1$ & 0.702 \\
\hline Mean procedure time (minute) & $96.8 \pm 54.5$ & $97.4 \pm 51.9$ & 0.966 \\
\hline Mean onset-to-final angiography time (minute) & $344.1 \pm 100.7$ & $325.3 \pm 87.9$ & 0.447 \\
\hline Successful reperfusion (\%) & $20 / 43(46.5)$ & $7 / 27(25.9)$ & 0.085 \\
\hline Hemorrhagic transformation, $\mathrm{n}(\%)$ & & & 0.006 \\
\hline None & $39 / 58(67.2)$ & $16 / 34(47.1)$ & \\
\hline $\mathrm{HI}-1$ & $6 / 58(10.3)$ & 0/34 (0) & \\
\hline $\mathrm{HI}-2$ & 2/58 (3.4) & $4 / 34(11.8)$ & \\
\hline $\mathrm{PH}-1$ & $6 / 58(10.3)$ & $3 / 34(8.8)$ & \\
\hline $\mathrm{PH}-2$ & $5 / 58(8.6)$ & $11 / 34(32.4)$ & \\
\hline Subarachnoid hemorrhage & $3 / 41(6.8)$ & $3 / 27(11.1)$ & 0.667 \\
\hline Median infarct volume $48-72 \mathrm{hr}, \mathrm{mL}$ (IQR) & $81(38-192)$ & $414(193-540)$ & $<0.001$ \\
\hline Median NIHSS at 1 day (IOR) & $15.5(12-21)$ & $22.5(19-33)$ & $<0.001$ \\
\hline Median NIHSS at discharge (IQR) & $11(7-20)$ & $42(20-42)$ & $<0.001$ \\
\hline Favorable outcome at 3 months & $26 / 58(44.8)$ & 3/34 (8.8) & $<0.001$ \\
\hline Mortality at 3 months & $14 / 58(24.1)$ & $23 / 34(67.6)$ & $<0.001$ \\
\hline
\end{tabular}

STO, simple internal carotid artery terminus occlusion; CTO, complex internal carotid artery terminus occlusion; CT, computed tomography; HI, hemorrhagic infarction; $\mathrm{PH}$, parenchymal hematoma; IQR, interquartile range; NIHSS, National Institute of Health Stroke Scale.

Table 3. Factors associated with a favorable outcome in patients with acute internal carotid artery terminus occlusion

\begin{tabular}{|c|c|c|c|c|}
\hline \multirow{2}{*}{ Variables } & \multicolumn{2}{|c|}{ Model 1} & \multicolumn{2}{|c|}{ Model 2} \\
\hline & OR $(95 \% \mathrm{Cl})$ & $P$ & $\mathrm{OR}(95 \% \mathrm{Cl})$ & $P$ \\
\hline Age $<80$ years & $2.52(0.55-11.61)$ & 0.235 & $2.89(0.18-46.61)$ & 0.455 \\
\hline Male sex & $3.01(0.95-9.54)$ & 0.062 & 3.99 (0.78-20.48) & 0.097 \\
\hline NIHSS score $<15$ on admission & $4.50(1.28-15.80)$ & 0.019 & $2.64(0.45-15.56)$ & 0.285 \\
\hline Diabetes mellitus & $0.26(0.05-1.28)$ & 0.098 & $0.20(0.02-2.05)$ & 0.177 \\
\hline STO & $6.11(1.55-24.04)$ & 0.010 & $11.16(1.09-114.41)$ & 0.042 \\
\hline Successful reperfusion & - & - & $1.35(0.28-6.56)$ & 0.713 \\
\hline
\end{tabular}

OR, odds ratio; $\mathrm{Cl}$, confidence interval; NIHSS, National Institute of Health Stroke Scale; STO, simple internal carotid artery terminus occlusion.

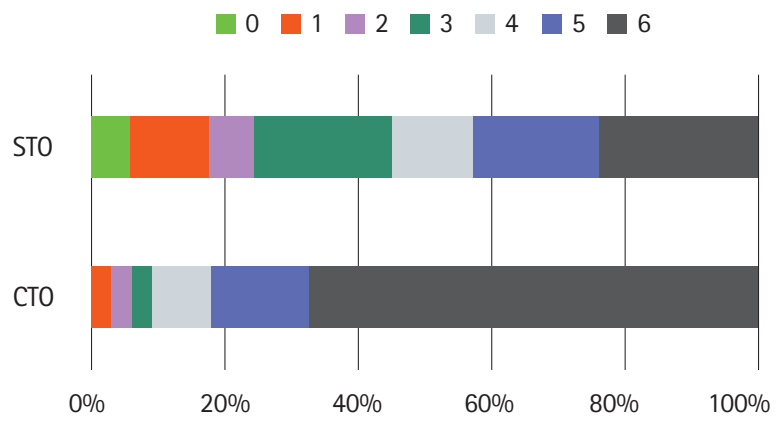

Figure 3. The modified Rankin Scale score at 3 months according to the occlusion pattern. More patients experiencing good outcomes and fewer patients experiencing poor outcomes were observed in the simple internal carotid artery (ICA) terminus occlusion (STO) group. Conversely, most patients in the complex ICA terminus occlusion (CTO) group were severely disabled or dead after 3 months.

matoma type 2 was lower ( $8.6 \%$ vs. $32.4 \%, P=0.006$ ). The median infarct volume at approximately 3 days was significantly higher in the CTO group (median, 81 vs. $414 \mathrm{~mL}, P<0.001$ ). The median NIHSS scores at discharge were 11 and 42 in the STO and CTO groups, respectively $(P<0.001)$. Favorable outcomes at 3 months were more frequently reported $(44.8 \%$ vs. $8.8 \%, P<0.001)$ and the 3-month mortality rate was significantly lower in the STO group ( $24.1 \%$ vs. $67.6 \%, P<0.001$ ) (Figure 3 ). Multivariable analysis demonstrated that the STO pattern was an independent predictor of favorable outcome at 3 months (Table 3).

\section{Discussion}

The principal finding of our study is that major differences in clinical outcome are apparent when ICA terminus occlusions are classified by circle of Willis involvement. CTO with compromised Willisian collateral flow had a very poor prognosis, whereas STO that spared the Willisian collateral flow had a significantly greater proportion of favorable outcomes with reperfusion treatments. We propose that ICA terminus occlusions should be divided into CTO and STO based on the degree of Willisian collateral circulation supplying the affected middle cerebral artery territory.

Our pragmatic treatment strategy resulted in favorable out- 
comes in STO, suggesting that this group is ideal for endovascular treatment. Conversely, the current study also highlights the detrimental effects of multiple occlusions or poor Willisian collaterals in some ICA occlusions. The baseline extent of early ischemic changes with NCCT ASPECTS and hypocontrast with PCCT ASPECTS was significantly greater in patients with $\mathrm{CTO}$, and the final infarct volume almost 5 times larger than in STO patients. Most authors suggest that aggressive revascularization treatment should not be performed if the initial core volume is too large ${ }^{19,20}$ hence, patients with CTO beyond a very early time window for reperfusion may represent poor candidates for such therapies in clinical trials and routine practice.

The importance of Willisian collaterals on tissue fate in the setting of terminal ICA occlusion has been studied relatively little compared to the leptomeningeal collaterals. The degree of leptomeningeal collaterals based on conventional angiography is known to predict outcome with reperfusion treatment. ${ }^{21}$ Regarding Willisian collaterals, conventional angiography requires cannulation of the contralateral carotid and posterior circulation. This is impractical during endovascular treatment as it would add unnecessary time delays to reperfusion. Similarly, Willisian collaterals have not been studied extensively with CTA in the past. ${ }^{22}$ Several CTA studies have examined the relationship between leptomeningeal collateral flow and outcome with reperfusion treatment. ${ }^{14,23}$ In the current study, CTA- and PCCT-colASPECTS grading indicated significantly poorer leptomeningeal collaterals in the CTO group. Combining CTA circle of Willis evaluation to classify ICA terminus occlusion and CTA-based leptomeningeal collateral grading may be the most comprehensive approach to fully evaluate collateral flow to the ischemic hemisphere, especially in the setting of ICA occlusion.

Recent clinical trials have shown that the outcomes of ICA terminus occlusions might be improved by endovascular treatment. The Interventional Management of Stroke III trial examined an ICA+M1 occlusion subgroup identified by CTA prior to randomization and showed a higher rate of recanalization at 24 hours and trend toward better outcomes with combined endovascular+IV rtPA treatment vs. IV rtPA treatment alone..$^{24}$ In a phase 3 randomized controlled trial, ESCAPE, the clinical outcomes for ICA occlusions were much better in patients treated by endovascular treatment up to 12 hours from symptom onset. ${ }^{25}$ This study used an ASPECTS score cutoff below 6 or no/ poor collaterals by multiphase CTA, which likely excluded many CTO patients. ${ }^{26}$ Patient selection can also be determined by CT or magnetic resonance perfusion, which was the case in both the EXTEND-IA and SWIFT PRIME trials. ${ }^{27,28}$ Both trials excluded large cores defined by long Tmax delays, also likely to exclude many CTO patients. ${ }^{29}$ Nevertheless, evaluation of circle of Willis flow may still have a role to distinguish carotid terminus occlusion types for prognosis and endovascular decision-making since single-phase CTA remains the most commonly used modality among most endovascular hospitals.

The present study has some limitations. It was a retrospective single-center study, and ICA terminus occlusions constitute only a small portion of all cerebral arterial occlusions causing acute ischemic stroke. The treatment strategy was heterogeneous, including both the previous and currently preferred endovascular treatment methods. Recent new-generation mechanical thrombectomy devices are more effective for recanalization and may produce better clinical outcomes. ${ }^{25,27,30}$ Nevertheless, there were no strict imaging criteria for patient selection in the earlier period of endovascular treatment; therefore, many CTO cases who undertook endovascular treatment were included in our study population. Currently, imaging criteria using multiphase CTA or multimodal magnetic resonance imaging are applied to differentiate patients with poor prognosis and thus, some CTO patients can be excluded using these protocols. ${ }^{31}$ In this situation, potential selection bias exists while analyzing clinical outcomes of CTO patients. Again, our method to differentiate CTO patients by CTA is simpler than multiphase or multimodal imaging protocols. However, further study should be performed to determine how СTO pattern influences collateral grading using multiphase CTA or ischemic core and penumbra mismatch using multimodal magnetic resonance imaging.

In conclusion, assessment of the configuration and patency of the circle of Willis with CTA may represent a non-invasive, practical, and time-saving method to estimate Willisian collateral status in ICA terminus occlusions and may hence be used to guide acute therapeutic intervention. Sub-classification of ICA terminus occlusions into STO and CTO can be utilized as a valuable early indicator of their prognosis. Among patients with acute ICA terminus occlusions, those with STO seem to be more amenable to active revascularization treatment. For a comparison with contemporary patient selection methods and treatment outcomes, recently-completed endovascular clinical trials should evaluate circle of Willis anatomy by CTA in case there are subgroups such as CTO patients that are very unlikely to benefit from endovascular treatment.

\section{References}

1. Saqqur M, Uchino K, Demchuk AM, Molina CA, Garami Z, Calleja $S$, et al. Site of arterial occlusion identified by transcranial Doppler predicts the response to intravenous thrombolysis for stroke. Stroke 2007;38:948-954.

2. Zaidat 00, Suarez Jl, Santillan C, Sunshine JL, Tarr RW, Paras 
$\mathrm{VH}$, et al. Response to intra-arterial and combined intravenous and intra-arterial thrombolytic therapy in patients with distal internal carotid artery occlusion. Stroke 2002;33:1821-1826.

3. Lee JS, Hong JM, Lee SJ, Joo IS, Lim YC, Kim SY. The combined use of mechanical thrombectomy devices is feasible for treating acute carotid terminus occlusion. Acta Neurochir (Wien) 2013;155:635-641.

4. Bhatia R, Hill MD, Shobha N, Menon B, Bal S, Kochar $P$, et al. Low rates of acute recanalization with intravenous recombinant tissue plasminogen activator in ischemic stroke: real-world experience and a call for action. Stroke 2010;41:2254-2258.

5. Lee JS, Hong JM, Kim EJ, Shin DH, Joo IS, Lim YC, et al. Comparison of the incidence of parenchymal hematoma and poor outcome in patients with carotid terminus occlusion treated with intra-arterial urokinase alone or with combined IV rtPA and intra-arterial urokinase. AJNR Am J Neuroradiol 2012;33: 175-179.

6. Arnold M, Nedeltchev K, Mattle HP, Loher TJ, Stepper F, Schroth $\mathrm{G}$, et al. Intra-arterial thrombolysis in 24 consecutive patients with internal carotid artery T occlusions. J Neurol Neurosurg Psychiatry 2003;74:739-742.

7. Bang OY, Saver JL, Kim SJ, Kim GM, Chung CS, Ovbiagele B, et al. Collateral flow averts hemorrhagic transformation after endovascular therapy for acute ischemic stroke. Stroke 2011; 42:2235-2239.

8. Chuang YM, Chan L, Lai YJ, Kuo KH, Chiou YH, Huang LW, et al. Configuration of the circle of Willis is associated with less symptomatic intracerebral hemorrhage in ischemic stroke patients treated with intravenous thrombolysis. J Crit Care 2013; 28:166-172.

9. Liebeskind DS, Flint AC, Budzik RF, Xiang B, Smith WS, Duckwiler GR, et al. Carotid I's, L's and T's: collaterals shape the outcome of intracranial carotid occlusion in acute ischemic stroke. J Neurointerv Surg 2015;7:402-407.

10. Osborn A. Diagnostic Cerebral Angiography. 2nd ed. Philadelphia: Lippincott Williams \& Wilkins, 1999.

11. Brott T, Adams HP Jr, Olinger CP, Marler JR, Barsan WG, Biller $J$, et al. Measurements of acute cerebral infarction: a clinical examination scale. Stroke 1989;20:864-870.

12. Higashida RT, Furlan AJ, Roberts H, Tomsick T, Connors B, Barr $J$, et al. Trial design and reporting standards for intra-arterial cerebral thrombolysis for acute ischemic stroke. Stroke 2003; 34:e109-e137.

13. Adams HP Jr, Bendixen BH, Kappelle $\sqcup$, Biller J, Love BB, Gordon $\mathrm{DL}$, et al. Classification of subtype of acute ischemic stroke. Definitions for use in a multicenter clinical trial. TOAST. Trial of Org 10172 in Acute Stroke Treatment. Stroke 1993;24:35-41.

14. Barber PA, Demchuk AM, Zhang J, Buchan AM. Validity and reliability of a quantitative computed tomography score in predicting outcome of hyperacute stroke before thrombolytic therapy. ASPECTS Study Group. Alberta Stroke Programme Early CT Score. Lancet 2000;355:1670-1674.

15. Choi JY, Kim EJ, Hong JM, Lee SE, Lee JS, Lim YC, et al. Conventional enhancement CT: a valuable tool for evaluating pial collateral flow in acute ischemic stroke. Cerebrovasc Dis 2011; 31:346-352.

16. Hacke $W_{1}$ Kaste $M$, Fieschi $C$, Toni $D$, Lesaffre $E$, von Kummer $R$, et al. Intravenous thrombolysis with recombinant tissue plasminogen activator for acute hemispheric stroke. The European Cooperative Acute Stroke Study (ECASS). JAMA 1995;274: 1017-1025.

17. Oppenheim C, Samson $Y$, Manaï $R$, Lalam $T$, Vandamme $X$, Crozier $S$, et al. Prediction of malignant middle cerebral artery infarction by diffusion-weighted imaging. Stroke 2000;31:21752181.

18. Zaidat 00, Yoo AJ, Khatri P, Tomsick TA, von Kummer R, Saver $J L$, et al. Recommendations on angiographic revascularization grading standards for acute ischemic stroke: a consensus statement. Stroke 2013;44:2650-2663.

19. Albers GW, Thijs VN, Wechsler L, Kemp S, Schlaug G, Skalabrin

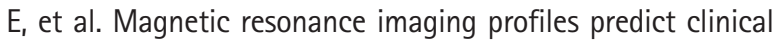
response to early reperfusion: the diffusion and perfusion imaging evaluation for understanding stroke evolution (DEFUSE) study. Ann Neurol 2006;60:508-517.

20. Davis SM, Donnan GA, Parsons MW, Levi C, Butcher KS, Peeters $A$, et al. Effects of alteplase beyond $3 \mathrm{~h}$ after stroke in the Echoplanar Imaging Thrombolytic Evaluation Trial (EPITHET): a placebo-controlled randomised trial. Lancet Neurol 2008;7: 299-309.

21. Christoforidis GA, Mohammad Y, Kehagias D, Avutu B, Slivka AP. Angiographic assessment of pial collaterals as a prognostic indicator following intra-arterial thrombolysis for acute ischemic stroke. AJNR Am J Neuroradiol 2005;26:1789-1797.

22. Maas MB, Lev $M H, A y H$, Singhal $A B$, Greer DM, Smith WS, et al. Collateral vessels on CT angiography predict outcome in acute ischemic stroke. Stroke 2009;40:3001-3005.

23. Tan JC, Dillon WP, Liu S, Adler F, Smith WS, Wintermark M. Systematic comparison of perfusion-CT and CT-angiography in acute stroke patients. Ann Neurol 2007;61:533-543.

24. Demchuk AM, Goyal M, Yeatts SD, Carrozzella J, Foster LD,

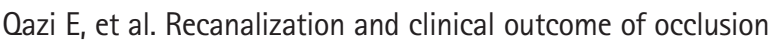
sites at baseline CT angiography in the Interventional Management of Stroke III Trial. Radiology 2014;273:202-210.

25. Goyal M, Demchuk AM, Menon BK, Eesa M, Rempel JL, Thornton $J$, et al. Randomized assessment of rapid endovascular treatment of ischemic stroke. N Engl J Med 2015;372:1019-1030. 
26. Demchuk AM, Goyal M, Menon BK, Eesa M, Ryckborst KJ, Kamal $\mathrm{N}$, et al. Endovascular treatment for Small Core and Anterior circulation Proximal occlusion with Emphasis on minimizing CT to recanalization times (ESCAPE) trial: methodology. Int J Stroke 2015;10:429-438.

27. Campbell BC, Mitchell PJ, Kleinig TJ, Dewey HM, Churilov L, Yassi $\mathrm{N}$, et al. Endovascular therapy for ischemic stroke with perfusion-imaging selection. N Engl J Med 2015;372:1009-1018.

28. Saver JL, Goyal M, Bonafe A, Diener HC, Levy El, Pereira VM, et al. Stent-retriever thrombectomy after intravenous t-PA vs. t-PA alone in stroke. N Engl J Med 2015;372:2285-2295.
29. Lin L, Bivard A, Parsons MW. Perfusion patterns of ischemic stroke on computed tomography perfusion. J Stroke 2013;15: 164-173.

30. Berkhemer OA, Fransen PS, Beumer D, van den Berg LA, Lingsma HF, Yoo AJ, et al. A randomized trial of intraarterial treatment for acute ischemic stroke. N Engl J Med 2015;372:11-20.

31. Lee JS, Demchuk AM. Choosing a hyperacute stroke imaging protocol for proper patient selection and time efficient endovascular treatment: lessons from recent trials. J Stroke 2015; $17: 221-228$ 